\title{
Optimal Gabor Filters for Steganalysis of Content-Adaptive J PEG Steganography
}

\author{
Xiaofeng Song ${ }^{1,2,3}$, Fenlin Liu ${ }^{1,3}$, Liju Chen ${ }^{2}$, Chunfang Yang ${ }^{1,3}$, Xiangyang Luo ${ }^{1,3}$ \\ ${ }^{1}$ Zhengzhou Science and Technology Institute \\ Zhengzhou 450001, Henan, China \\ ${ }^{2}$ Xi'an Communication Institute, Xi'an 710106, China \\ ${ }^{3}$ State key Laboratory of Mathematical Engineering and Advanced Computing \\ Zhengzhou, Henan 450001, China \\ [e-mail: xiaofengsong@sina.com, chunfangyang@126.com] \\ *Corresponding author: Xiaofeng Song, Chunfang Yang
}

Received July 20, 2016; revised September 9, 2016; accepted November 28, 2016;

published January 31, 2017

\begin{abstract}
The existing steganalysis method based on 2D Gabor filters can achieve a competitive detection performance for content-adaptive JPEG steganography. However, the feature dimensionality is still high and the time-consuming of feature extraction is relatively large because the optimal selection is not performed for 2D Gabor filters. To solve this problem, a new steganalysis method is proposed for content-adaptive JPEG steganography by selecting the optimal 2D Gabor filters. For the proposed method, the 2D Gabor filters with different parameter settings are generated first. Then, the feature is extracted by each 2D Gabor filter and the corresponding detection accuracy is used as the measure for filter selection. Next, some 2D Gabor filters are selected by a greedy strategy and the steganalysis feature is extracted by the selected filters. Last, the ensemble classifier is used to assemble the proposed steganalysis feature as well as the final steganalyzer. The experimental results show that the steganalysis feature extracted by the selected optimal 2D Gabor filters also can achieve a competitive detection performance while the feature dimensionality is reduced greatly.
\end{abstract}

Keywords: Content-adaptive steganography, JPEG, steganalysis, 2D Gabor filter, optimal selection

This work was supported by the National Natural Science Foundation of China (No. 61272489, 61379151, 61302159 and 61602508), the Excellent Youth Foundation of Henan Province of China (Grant No. 144100510001), and the Natural Science Basic Research Plan in Shaanxi Province of China (No.2014JM2-6103). 


\section{Introduction}

Steganography and steganalysis of JPEG image are always the popular research topics in information hiding. JPEG steganography tries to preserve the image statistics while the steganalysis techniques need to find the other image statistics that can capture the embedding changes. The steganography and steganalysis techniques promote each other and accelerate the development of information hiding research. Compared with the typical JPEG steganography algorithms such as Jsteg [1], OutGuess [2], MB [3], F5 [4], nsF5 [5], PQ (perturbed quantization) [6] and MME (modified matrix encoding) [7], the latest JPEG steganography algorithms such as PQt (texture-adaptive PQ) and PQe [5] (energy-adaptive PQ), MOD (Model Optimized Distortion) [8], NPQ (Normalized Perturbed Quantization) [9], EBS (Entropy Block Steganography) [10], UED (Uniform Embedding Distortion) [11], J-UNIWARD (JPEG UNIversal WAvelet Relative Distortion) [12], SI-UNIWARD (Side-Informed UNIWARD) [12] and so on are all content-adaptive. The content-adaptive JPEG steganography algorithms often define a heuristic embedding distortion function and then assign a cost of changing each cover element. The given messages are embedded by syndrome-coding methods, such as the Syndrome-Trellis Codes (STCs) [13]. For the embedding changes are constrained to the complex textures or noisy areas, the content-adaptive JPEG steganography can achieve the better steganographic security. The old steganalysis methods almost $[14,15,16]$ often have poor detection performances.

For content-adaptive JPEG steganography, some new steganalysis methods have been proposed in recent years. These methods often construct the detectors by classifiers trained on the statistical features of cover and stego images. Therefore, the feature extraction is important for steganalysis of content-adaptive JPEG steganography. According to the feature extraction source, these steganalysis methods can be divided into two types. For the first type, the features are extracted from quantized DCT coefficients. For example, in [17], for MOD steganography, the distortion function optimized to maximize security has been overtrained to an incomplete cover model, therefore the inter-block co-occurrences feature beyond the optimized model is proposed for the detection performance. In [18], the principle of PQt and PQe steganography is analyzed and the enhanced histogram feature is proposed to improve the detection performance. In [19], in order to capture the embedding changes more comprehensively, a rich model of DCT coefficients in a JPEG files is proposed. For the second type, the features are extracted from the spatial-domain representation of the JPEG image. For example, in [20], by projecting neighboring residual samples onto a set of random vectors, the PSRM (Projection Spatial Rich Model) feature is proposed. PSRM feature takes the first-order statistic (histogram) of the projections as the feature instead of forming the co-occurrence matrix. In [21], the DCTR (Discrete Cosine Transform Residual) feature which utilizes 64 kernels of the discrete cosine transform is proposed. DCTR can achieve the better detection performances for content-adaptive JPEG steganography while preserving relatively low complexity. In [22], the PHARM (Phase-Aware Projection Model) is proposed by utilizing the JPEG image pixel residuals and their phase w.r.t. the $8 \times 8$ grid. The PHARM feature can get better detection accuracy than DCTR for content-adaptive JPEG steganography. In [23], a novel steganalysis feature is proposed based on 2D Gabor filters. The 2D Gabor filters can describe the image texture characteristics from different scales and orientations, so the proposed feature GH (Gabor Histogram) improve the detection accuracy for content-adaptive JPEG steganograpy which often constrains the embedding changes to complex textures. After 
feature extraction, in [17], the final detector is constructed by SVM (Support Vector Machine), in [18-23], the detectors are all constructed by ensemble classifier [24] which is designed for high-dimensional feature.

For the above steganalysis features, the 2D Gabor filters-based feature proposed in [23] achieves the competitive detection performances for content-adaptive JPEG steganography. However, the feature dimensionality is still high and the time consuming is relatively large because the Optimal Selection is not performed for 2D Gabor filters. In other words, the "good" 2D Gabor filters should be picked up for feature extraction. As we know, the detection accuracies of the steganalysis features extracted by different 2D Gabor filters are often different. By selecting the 2D Gabor fiters, more compact steganalysis feature can be got while the feature dimensionality will be reduced and the detection accuracy may be improved. Based on the above idea, a feature extraction method is proposed for steganalysis of content-adaptive JPEG steganography by selecting 2D Gabor filters. Firstly, the design of 2D Gabor filter is introduced and the effects of the parameter settings for steganalysis are discussed. Then, the selection of 2D Gabor filters is studied and the corresponding feature extraction method is proposed for steganalysis of content-adaptive JPEG steganography.

The rest of this paper is arranged as follows. Section 2 describes the image decomposition by 2D Gabor filters, Section 3 describes the proposed feature extraction method, and Section 4 shows the experimental results and analyses. Lastly, the conclusions and future works are given in Section 5.

\section{Image Decomposition Using 2D Gabor Filters}

2D Gabor filter can achieve optimal joint localization properties in the spatial domain and in the spatial frequency domain [25]. For the desirable characteristics of spatial locality and orientation selectively, 2D Gabor filters are widely used for texture image classification [26], texture image segmentation [27], iris recognition [28] and face recognition [29], etc. For steganography and steganalysis, in [30], the 2D Gabor filter is used for the definition of distortion function of content-adaptive steganography and the better steganographic security is achieved than db8 wavelet; in [23], the 2D Gabor filter is used for steganalysis of content-adaptive JPEG steganography and the better detection performances are got. In this section, the image decomposition using 2D Gabor filters is discussed firstly and then the properties of filtered image coefficients are analyzed.

\subsection{Decomposing image by 2D Gabor filters}

When the 2D Gabor filters are used for steganalysis, the image should be filtered by 2D Gabor filters firstly and then the feature extraction can be performed. The 2D Gabor filtering for image is that an input image $I(x, y)$ is convolved with a 2D Gabor function $g(x, y)$ to obtain a Gabor feature image $u(x, y)$ as follows:

$$
u(x, y)=\iint_{\Omega} I(\xi, \eta) g(x-\xi, y-\eta) d \xi d \eta
$$

where, $(x, y) \in \Omega, \Omega$ denotes the set of image points.

In this paper, the 2D Gabor function $g(x, y)$ in Eq. (1) uses the following family of Gabor functions [23, 30, 31], it is a product of a Gaussian and a cosine function.

$$
g_{\lambda, \theta, \varphi}(x, y)=e^{-\left(\left(x^{\prime 2}+\gamma^{2} y^{\prime 2}\right) / 2 \sigma^{2}\right)} \cos \left(2 \pi \frac{x^{\prime}}{\lambda}+\varphi\right)
$$


where, $x^{\prime}=x \cos \theta+y \sin \theta, y^{\prime}=-x \sin \theta+y \cos \theta, \sigma=0.56 \lambda, \gamma=0.5$.

The parameters of 2D Gabor function in Eq. (2) are important for filter design. In Table 1, a simple description of the parameters of 2D Gabor function is given.

Table 1. A description of parameters of 2D Gabor function

\begin{tabular}{cr}
\hline Parameter & Description \\
\hline$\sigma$ & Standard deviation sigma of the Gaussian factor. \\
\hline$\theta$ & Orientation of the normal to the parallel stripes of the Gabor function. \\
\hline$\gamma$ & Phase offset of the cosine factor of the Gabor function. \\
\hline$\gamma$ & Ellipticity of the Gaussian factor. \\
\hline$\lambda$ & Wavelength of the cosine factor of the Gabor function.
\end{tabular}

For image filtering, the 2D Gabor function in Eq. (2) must be discretized to get the corresponding 2D Gabor filters. The main steps for function discretization can be described as follows.

1) Suppose the size of $2 \mathrm{D}$ Gabor filter is $M \times N$, the range of $x$ and $y$ in Eq. (2) is set to $\left\{-\frac{M-1}{2}, \cdots, \frac{M-1}{2}\right\}$ and $\left\{-\frac{N-1}{2}, \cdots, \frac{N-1}{2}\right\}$ respectively, and then the sampling points $(x, y)$ are got by 1 .

2) The parameters of 2D Gabor function given in Table $\mathbf{1}$ are set.

3) The 2D Gabor filters with different parameters are generated according to Eq. (2). Then, all the 2D Gabor filters are made zero mean by subtracting the kernel mean from all its elements to form high-pass filter.

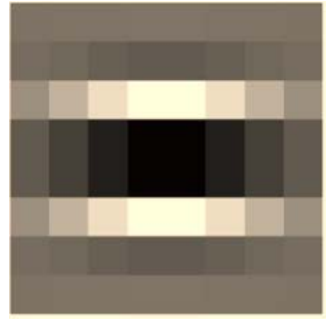

$\theta=0$

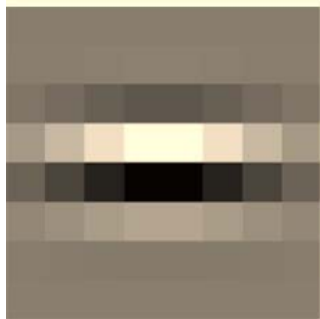

$\theta=0$

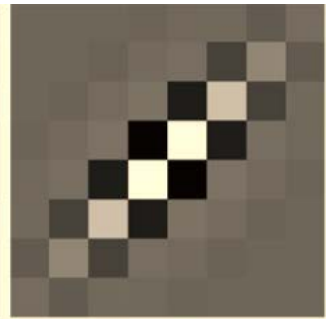

$\theta=\pi / 4$

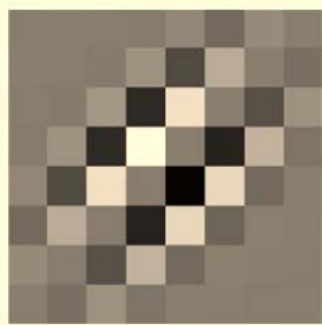

$\theta=\pi / 4$

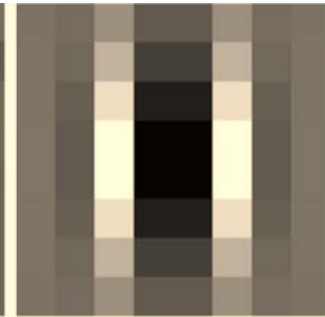

$\theta=2 \pi / 4$

(a)

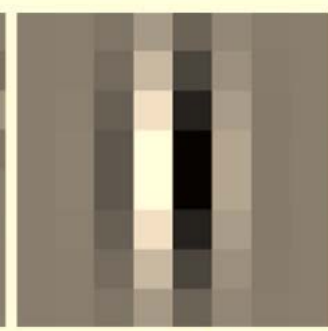

$\theta=2 \pi / 4$

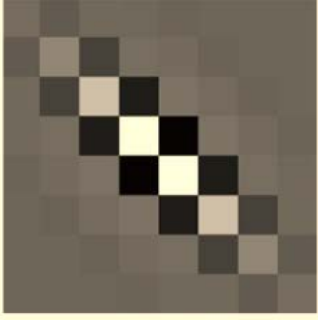

$\theta=3 \pi / 4$

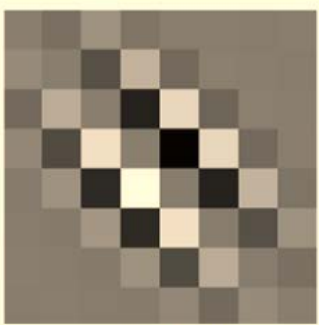

$\theta=3 \pi / 4$

(b)

Fig. 1. 2D Gabor filters with different parameter settings. (a) $\sigma=1, \varphi=0$; (b) $\sigma=1, \varphi=\pi / 2$.

In Fig. 1, the 2D Gabor filters with different parameter settings are shown and the size of the 2D Gabor filters is $8 \times 8$. In Fig. 1(a), the four 2D Gabor filters have different orientation parameters, the scale parameter $\sigma=1$ and phase offset parameter $\varphi=0$. In Fig. 1(b), the 
parameter $\varphi$ is set to $\pi / 2$. From Fig. 1, it can be seen that the 2D Gabor filter is centrosymmetric when $\varphi=0$ and the filter is anti-centrosymmetric when $\varphi=\pi / 2$. Moreover, when the orientation parameter $\theta$ is different, the 2D Gabor filter can act as different orientation filter.

For 2D Gabor filter, parameter $\sigma$ is also known as the scale parameter. The small $\sigma$ means high spatial resolution and the filtered image coefficients reflect image local properties at fine scale. On the contrary, the large $\sigma$ means low spatial resolution and the filtering coefficients reflect image local properties at coarse scale. For the relation between $\sigma$ and $\lambda$ is fixed, only the paramters $\sigma, \theta$ and $\varphi$ need to be determined when 2D Gabor filter is designed. The effects of the parameters $\sigma, \theta$ and $\varphi$ for steganalysis are analyzed as follows.

(1) The scale parameter $\sigma$ is a real number greater than 0 and the 2D Gabor filter with different scale parameters can capture the embedding changes in different spatial resolution. Therefore, 2D Gabor filters utilized for steganalysis feature extraction should have diverse and suitable scale parameters.

(2) The valid values of parameter $\theta$ are real numbers between 0 and $\pi$. The 2D Gabor filters with different orientation parameters can capture image local characteristic from different orientations. Therefore, different orientation parameters should be set for steganalysis feature extraction.

(3) The parameter $\varphi$ determines the symmetry of the concerned Gabor filter: $\varphi=0, \pi$, the filter is centrosymmetric; $\varphi=-\pi / 2, \pi / 2$, the filter is anti-centrosymmetric. To enrich the diversity of 2D Gabor filters, parameter $\varphi=0, \pi / 2$ are often considered at the same time.

\subsection{Properties of filtered image coefficients}

Before the JPEG image is filtered by 2D Gabor filter, the JPEG file should be decompressed to the spatial domain. In order to avoid any loss of information, the JPEG image should be decompressed without quantizing the pixel values to $\{0,1, \ldots, 255\}$. Let us suppose the decompressed JPEG image is denoted as $\mathbf{I}^{\prime}$, then the filtered image $\mathbf{U}^{s, l}=\mathbf{I}^{\prime} \star \mathbf{G}^{s, l}, \mathbf{G}^{s, l}$ specifies the $8 \times 8$ 2D Gabor filter in $s$ scale and $l$ orientation, ' $\star$ 'denotes a convolution without padding. Furthermore, suppose $\mathbf{B}^{(i, j)}$ denotes a $8 \times 8$ DCT basis pattern, $\mathbf{B}^{(i, j)}=\left(B_{m n}^{(i, j)}\right), 0 \leq m, n \leq 7,0 \leq i, j \leq 7$,

$$
B_{m n}^{(i, j)}=\frac{w_{i} w_{j}}{4} \cos \frac{\pi i(2 m+1)}{16} \cos \frac{\pi j(2 n+1)}{16}
$$

where, $w_{0}=1 / \sqrt{2}, w_{i}=1(i>0)$.

Then, the modification of DCT coefficient in mode $(i, j)$ of $8 \times 8$ DCT block will affect all the $8 \times 8$ pixels in the corresponding block, and an entire $15 \times 15$ neighborhood of values in $\mathbf{U}^{s, l}$. The values will be modified by "unit response" [21] expressed in Eq. (4).

$$
\mathbf{R}^{(i, j)(s, l)}=\mathbf{B}^{(i, j)} \otimes \mathbf{G}^{s . l}
$$

where, $\otimes$ denotes the full cross-correlation.

From Eq. (4), it can be seen that the unit response for the filtered image generated by the 2D Gabor filters is similar to the filtered image generated by the DCT kernel [21]. The only difference between them is the filter. However, note that the symmetry of unit response $R$ is 
different from the unit response in [21]. The absolute values of $R$ are centrosymmetric: $\left|R_{a, b}^{(s, l)(i, j)}\right|=\left|R_{-a, b}^{(s, l)(i, j)}\right|$ for all $0 \leq a, b \leq 7 \quad$ when indexing $R \in^{15 \times 15}$ with indices in $\{-7, \cdots,-1,0,1, \cdots, 7\}$.

In Fig. 2, two examples of unit responses caused by modifying the DCT coefficient in different modes are shown. From Fig. 2, it can be seen that the absolute values of unit response $R$ are centrosymmetric.

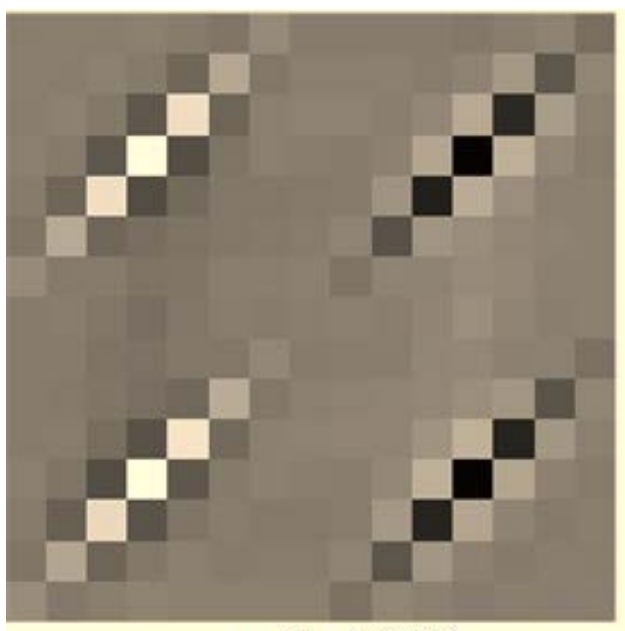

$\mathbf{R}^{(1, \pi / 4)(2,1)}$

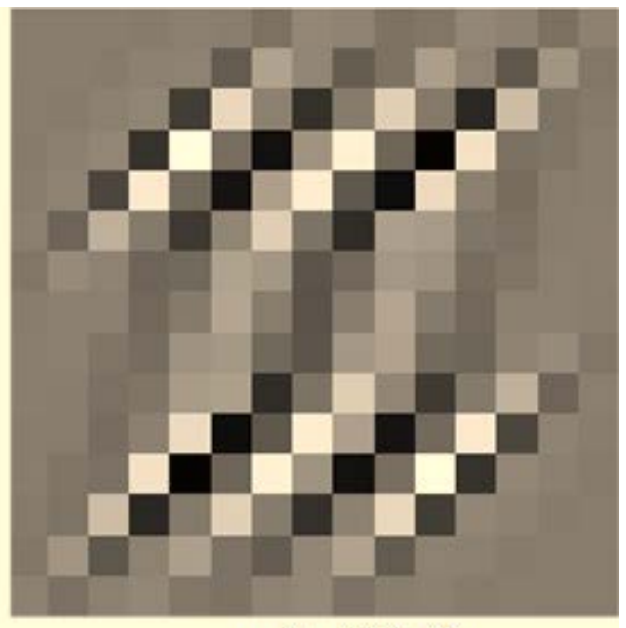

$\mathbf{R}^{(1, \pi / 4)(3,5)}$

Fig. 2. Two unit responses caused by embedding changes in DCT modes $(2,1)$ and $(3,5)$, where parameter $\varphi=0$.

We now look at how a particular value $u \in U^{s, l}$ is computed when the decompressed JPEG image is filtered by 2D Gabor filters. First, the filtered image $U^{s, l}$ is divided into $8 \times 8$ blocks. Next, as shown in Fig. 3, suppose A,B,C,D denote the four sampling points by $8, u$ is at position $(a, b)$ with respect to the upper left neighbor (A), $0 \leq a, b \leq 7$ (i.e., $(a=3, b=2)$ in Fig. 3). Then, the relative positions of $u$ with respect to the other three neighbors ( B,C,D) are $(a, b-8),(a-8, b)$, and $(a-8, b-8)$, respectively.

According to [21], filtered image coefficient $u$ can be denoted as

$$
\begin{aligned}
& u=\sum_{i=0}^{7} \sum_{j=0}^{7} Q_{i j}\left[A_{i j} R_{a, b}^{(s, l)(i, j)}+B_{i j} R_{a, b-8}^{(s, l)(i, j)}\right. \\
& \left.+C_{i j} R_{a-8, b}^{(s, l)(i, j)}+D_{i j} R_{a-8, b-8}^{(s, l)(i, j)}\right]
\end{aligned}
$$

where $A_{i j}, B_{i j}, C_{i j}$, and $D_{i j}$ specify the DCT coefficients of the corresponding four blocks in Fig. 3 when each $8 \times 8$ pixel block is replaced with a block of quantized DCT coefficients, $(i, j)$ specifies the $(i, j)$-th DCT mode, and $Q_{i j}$ is the quantization step in DCT mode $(i, j)$.

Value $u$ also can be written as a projection of 256 dequantized DCT coefficients from four adjacent blocks from the JPEG file using projection vector $P_{a, b}^{(s, l)}$ as follows: 


$$
u=\left(\begin{array}{c}
Q_{00} A_{00} \\
\vdots \\
Q_{77} A_{77} \\
Q_{00} B_{00} \\
\vdots \\
Q_{77} B_{77} \\
\vdots \\
Q_{00} D_{00} \\
\vdots \\
Q_{77} D_{77}
\end{array}\right)^{\left(\begin{array}{c}
R_{a, b}^{(s, l)(1,1)} \\
\vdots \\
R_{a, b}^{(s, l)(8,8)} \\
R_{a, b-8}^{(s, l)(1,1)} \\
\vdots \\
R_{a, b-8}^{(s, l)(8,8)} \\
\vdots \\
R_{a, b}^{(s, l)(1,1)} \\
\vdots \\
R_{a-8, b-8}^{(s, l)(8,8)}
\end{array}\right)} .
$$

If the unit response $R_{a, b}^{(s, l)(i, j)}$ is centrosymmetric, then the projection vectors satisfy the following symmetry

$$
\left|\mathrm{P}_{a, b}^{(s, l)}\right|=\left|\mathrm{P}_{a-8, b-8}^{(s, l)}\right|
$$

for all $i, j, a$, and $b$ when interpreting the arithmetic operations on indices as mod 8 .

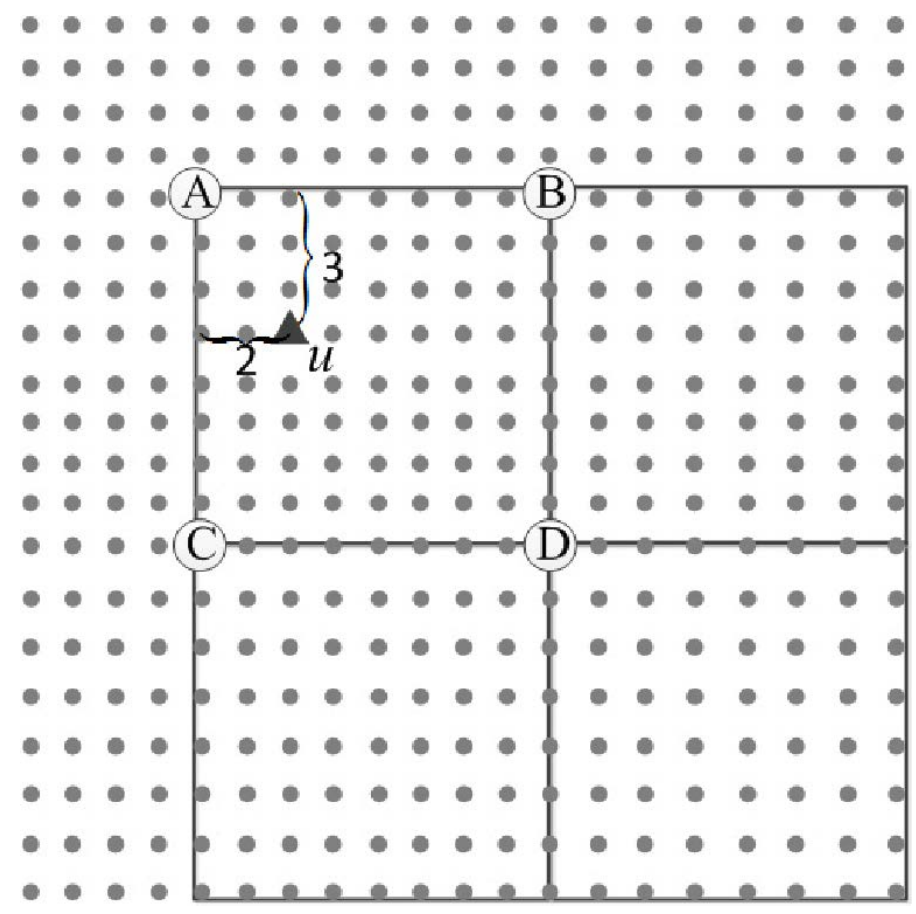

Fig. 3. Representation of filtered image coefficients $U^{s, l}$. The dots correspond to elements of $U^{s, l}$ and circles correspond to sampling points. 


\section{Feature Extraction by Optimal 2D Gabor Filters}

In literature [23], the 2D Gabor filters with different parameter settings are utilized for steganalysis feature extraction. However, the scale and orientation parameters are limited with respect to the whole parameter space. Although the detection accuracy can be improved by increasing 2D Gabor filters with different parameters, the feature dimensionality will be increased and the time consuming become large. Therefore, the optimal 2D Gabor filters should be selected for feature extraction. In this section, the 2D Gabor filters selection is studied firstly and then the feature extraction method is proposed.

\subsection{D Gabor filters selection}

When the 2D Gabor filters are selected, the 2D Gabor filters with different parameter settings are generated firstly. Then, the steganalysis feature is extracted by each filter and the evaluation measure is got. Lastly, the 2D Gabor filters are selected according to the evaluation measures. The detailed processes can be described as follows.

\section{(1) 2D Gabor filters generating}

The ranges of parameters $\sigma, \theta$ and $\varphi$ are set firstly, then multigroup parameters $(\sigma, \theta, \varphi)$ are generated randomly according to uniform distribution and the corresponding 2D Gabor filters are also generated. It should be noticed that the sizes of all the 2D Gabor filters are $8 \times 8$ because the size of DCT block of JPEG image is $8 \times 8$.

\section{(2) 2D Gabor filters evaluating}

To evaluate the 2D Gabor filters for steganalysis, the detection accuracy of the corresponding feature extracted by each 2D Gabor filter is used as the evaluation measure. The detailed process can be described as follows.

Step1: For each 2D Gabor filter $\mathbf{G}^{s, \theta, \varphi}$, the corresponding steganalysis feature is extracted according to the following steps.

1) The JPEG image is decompressed to spatial domain without quantizing the pixel values to $\{0,1, \cdots, 255\}$ to avoid any loss of information.

2) The decompressed JPEG image is convolved with each $2 \mathrm{D}$ Gabor filter $\mathbf{G}^{\sigma, \theta, \varphi}$, the filtered image is $\mathbf{U}^{\sigma, \theta, \varphi}$.

3) According to the 64 positions $(a, b)(0 \leq a \leq 7,0 \leq b \leq 7)$ in $8 \times 8$ image block, the filtered image $\mathbf{U}^{\sigma, \theta, \varphi}$ is subsampled by step size 8 to get 64 subimages $\mathbf{U}_{a, b}^{\sigma, \theta, \varphi}$ (As shown in Fig. 4).

4) For each subimage $\mathbf{U}_{a, b}^{\sigma, \theta, \varphi}$, the histogram feature is extracted by Eq. (8),

$$
\mathbf{h}_{a, b}^{\sigma, \theta, \varphi}(x)=\frac{1}{\left|\mathbf{U}_{a, b}^{\sigma, \theta, \varphi}\right|} \sum_{u \in \mathbf{U}_{a, b}^{\sigma, \theta, \varphi}}\left[Q_{T}(|u| / q)=x\right]
$$

where, $Q_{T}$ is a quantizer with integer centroids $\{0,1, \cdots, T\}, q$ denotes the quantization step, and $[P]$ is the Iverson bracket equal to 0 when the statement $P$ is false and 1 when $P$ is true.

5) According to the symmerty shown in Eq. (7), the features extracted in different positions can be merged to reduce feature dimensionality (As shown in Fig. 5). Therefore, all the histogram features of 64 subimages $\mathbf{U}_{a, b}^{\sigma, \theta, \varphi}$ are merged and combined to get the histogram feature of the filtered image $\mathbf{U}^{\sigma, \theta, \varphi}$. The dimensionality of histogram feature is $34 \times(T+1)$ for each filtered image $\mathbf{U}^{\sigma, \theta, \varphi}$. 
Step2: The detection accuracies of the steganalysis features are obtained by utilizing ensemble classifier.

Step3: The detection accuracies of the steganalysis features are used as the evaluation measures of the corresponding 2D Gabor filters.

\section{(3) 2D Gabor filters selecting}

For the selection of 2D Gabor filters, a simple forward selection strategy is applied. This strategy is greedy and the filters are selected sequentially one by one based on how much they improve the detection w.r.t. the union of those already selected. The first selected filter is the one with the best detection accuracy.

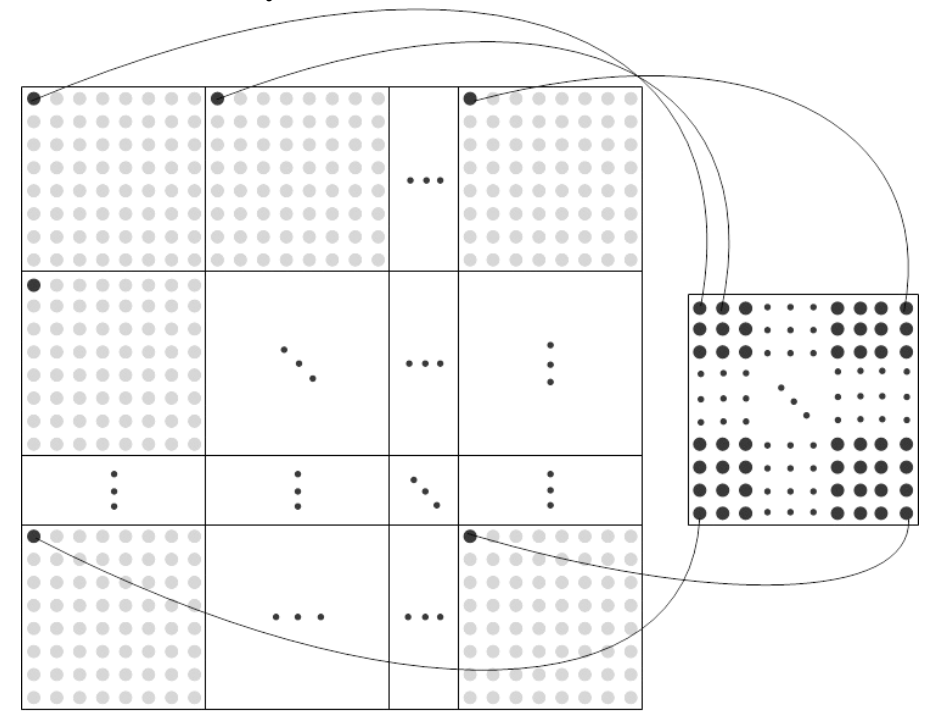

Fig. 4. Filtered image subsampling according to the 64 different positions $(a, b)$

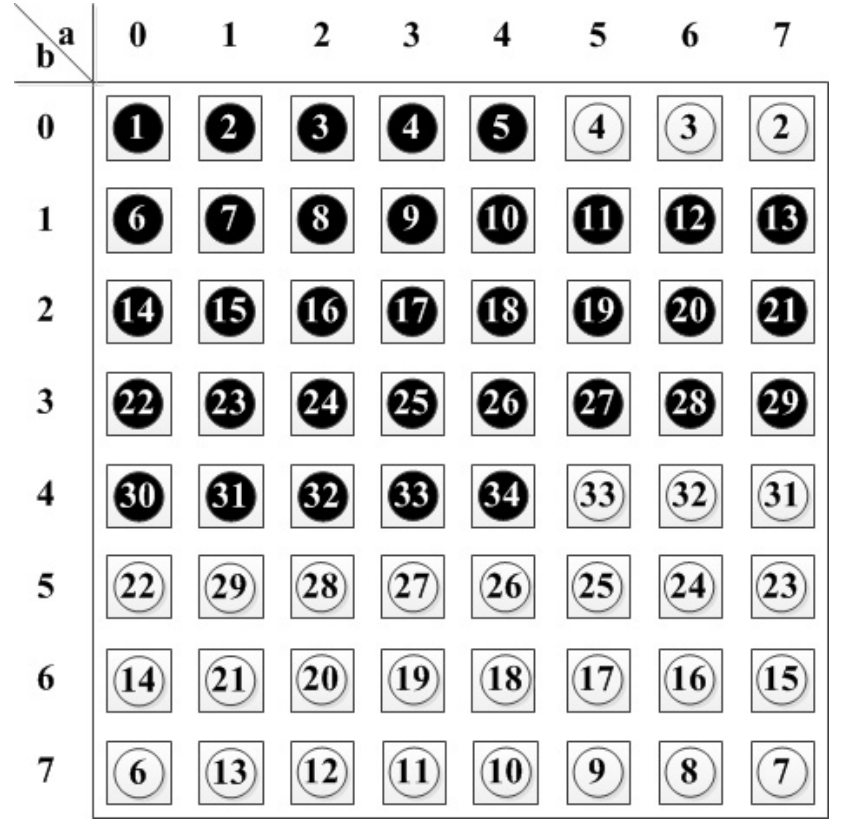

Fig. 5. The diagram of feature merging for each filtered image 


\subsection{Feature extraction}

Based on the above selection method for 2D Gabor filters, the feature extraction method for steganalysis of content-adaptive JPEG steganography is presented as follows.

Step1: A certain number of 2D Gabor filters are selected according to their detection accuracies.

Step2: For each selected 2D Gabor filter, the corresponding feature is extracted according to the method shown in Section 3.1.

Step3: All the features are combined to form the final steganalysis feature.

From all above, it can be seen that the key steps of the proposed steganalysis method is selection of 2D Gabor filters and feature extraction. Here, a simple example is used to explain the proposed steganalysis method. Suppose 1000 2D Gabor filters with different parameter settings have been generated, and then the corresponding steganalysis feature extracted by each 2D Gabor filter can be obtained according to Step 1. The feature dimensionality is $34 \times(T+1)$. Next, ensemble classfier is used to assemble each steganalysis feature and the detection accuracy is used as the evaluation measure of the corresponding 2D Gabor filter. According to the evaluation measures, suppose 100 2D Gabor filters with the best evaluation measures are selected. Then, the final steganalysis feature is extracted by the selected $1002 \mathrm{D}$ Gabor filters and the dimensionality is $100 \times 34 \times(T+1)$. Last, the ensemble classifier is used to evaluate the final steganalysis feature and as the final steganalyze.

\section{Experimental Results and Analysis}

\subsection{Image database and experimental setup}

In the experiments, the image database is BOSSbase 1.01 [32] containing 10000 grayscale $512 \times 512$ images with PGM format. For UED and J-UNIWARD steganography, all these grayscale images are converted into JPEG image with quality factor 75 and 95 respectively, and then the corresponding stego images are generated with payload $0.05,0.1,0.15,0.2,0.3$, $0.4,0.5$ bpac. For SI-UNIWARD steganography, the original grayscale images are used as precover images, and then the corresponding stego images are generated with payload from 0.05bpac to 0.5bpac when the grayscale images are compressed to JPEG image with quality factor 75 and 95 . Then, for each steganography algorithm and quality factor, there are one group cover images and seven group stego images, one group cover images and one group corresponding stego images are used as image samples for one payload. In all experiments, ensemble classifier [24] is used for the training and testing. The proportion of training set to

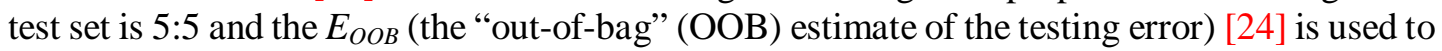
evaluate the detection performance of the steganalysis feature. The detection accuracy is the average value of ten duplicate experiments.

\subsection{Impact of filter parameter on detection accuracy}

In this subsection, the impact of 2D Gabor filter parameters on detection accuracy is discussed. In the experiments, the cove images are 10000 JPEG images obtained from Image database BOSSbase1.01 and the quality factor is 75 . The corresponding stego images are generated by J-UNIWARD and the payload is 0.3bpac. The steganalysis feature is extracted by single 2D Gabor filters with different parameter setting according to Eq. (8). 


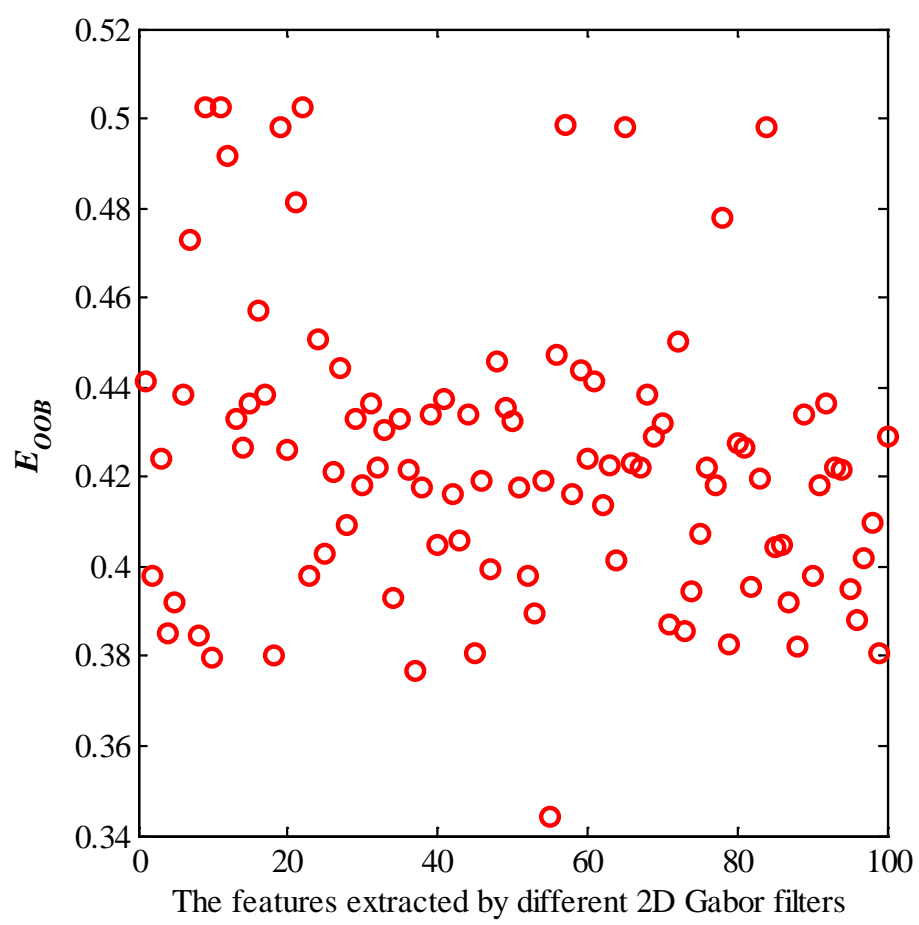

Fig. 6. The detection accuracies of the features extracted by different 2D Gabor filters

For the proposed steganalysis method, the threshold $T$ and quantization step $q$ should be determined for feature extraction. In [23], the settings of threshold $T$ and quantization step $q$ have been discussed and the appropriate values were selected. However, in [23], the quantization step $q$ is only given for quality factors 75 and 95 . In fact, the following linear fit can be adopted to obtain the proper value of $q$ for an arbitrary quality factor in the range of $50 \leq K \leq 99$ and arbitrary scale parameter $\sigma$.

$$
q=\sigma \cdot(107-K) / 8
$$

where $K$ denotes the quality factor, $50 \leq K \leq 99$.

In addition, in order to reduce feature dimensionality, the threshold $T$ is set to 2 and then the dimensionality of feature extracted by each 2D Gabor filter is 102.

In Fig. 6, the detection accuracies of the different steganalysis feature extracted by $1002 \mathrm{D}$ Gabor filters are given. These 2D Gabor filters are generated randomly and the ranges of parameters $\sigma, \theta$ and $\varphi$ are set as: $0.2 \leq \sigma \leq 2,0 \leq \theta<\pi, \varphi \in\{0, \pi / 2\}$.

From Fig. 6, it can be seen that the detection accuracies of the steganalysis features extracted by different 2D Gabor filters are diverse. The lowest $\mathrm{E}_{\mathrm{OOB}}$ is only $34.41 \%$ while the highest $\mathrm{E}_{\text {Оов }}$ is $49.85 \%$. Therefore, the $2 \mathrm{D}$ Gabor filters related with the steganalysis features which have high detection accuracies should be selected for feature extraction. The selection of 2D Gabor filters can not only reduce the dimensionality of the final steganalysis feature, but also reduce the time needed for feature extraction.

\subsection{Effect of 2D Gabor filters selection}

To evaluate the effect of 2D Gabor filters selection for steganalysis, the following experiment is performed for J-UNIWARD steganography. The cover images are the 10000 
JPEG images generated from BOSSbase 1.01 and the quality factor is 75 . The stego image is generated by J-UNIWARD steganography with 0.3 bpac. According to the selection method in section 3.1, the ranges of $\sigma, \theta$ and $\varphi$ are set as: $0.2 \leq \sigma \leq 2,0 \leq \theta<\pi, \varphi \in\{0, \pi / 2\}$ firstly. Then, 1000 groups $(\sigma, \theta, \varphi)$ are generated and the corresponding 2D Gabor filters are generated at the same time. Next, the feature is extracted according to Eq. (8) where the threshold $T=2$ and quantization step $q=\sigma \cdot(107-K) / 8$ ( $K$ denotes the quality factor). Last, the detection accuracy of the feature is got and the Gabor filters are selected one by one.

In Fig. 7, the distribution of detection accuracies $\left(\mathrm{E}_{\mathrm{OOB}}\right)$ of the features extracted by 1000 2D Gabor filters is shown. From Fig. 7, it can be seen that the $\mathrm{E}_{\text {Оов }}$ of the features extracted by some 2D Gabor filters are relatively low while the $\mathrm{E}_{\mathrm{OOB}}$ of the features extracted by some other 2D Gabor filters are relatively high. In other words, the detection accuracies of different 2D Gabor filters are diverse.

In Fig. 8, the effect of 2D Gabor filters selection for steganalysis is presented. From Fig. 8, it can be seen that the $\mathrm{E}_{\mathrm{OOB}}$ is decreased continually with the number of the selected 2D Gabor filters increases. Moreover, the $\mathrm{E}_{\text {оов }}$ remain almost constant when enough 2D Gabor filters have been selected. In addition, it should be noticed that the $\mathrm{E}_{\mathrm{OOB}}$ of the feature with 6936 dimensions $(68 \times 34 \times(2+1)=6936)$ extracted by 68 selected $2 \mathrm{D}$ Gabor filters is $17.40 \%$ while the $\mathrm{E}_{\mathrm{OOB}}$ of the feature with 17000 dimensions extracted by 256 2D Gabor filters in [23] is only $18.47 \%$. Therefore, the selection of 2D Gabor filters not only can reduce the feature dimensionality but also can improve the detection performance to some extent.

In Fig. 9, the distributions of parameters $\sigma, \theta$ and $\varphi$ of the selected 2D Gabor filters are shown. From Fig. 9, it can be seen that the parameters of the selected 2D Gabor filters are diverse. This is possibly because that the diverse 2D Gabor filters can enhance the effectiveness of the steganalysis feature.

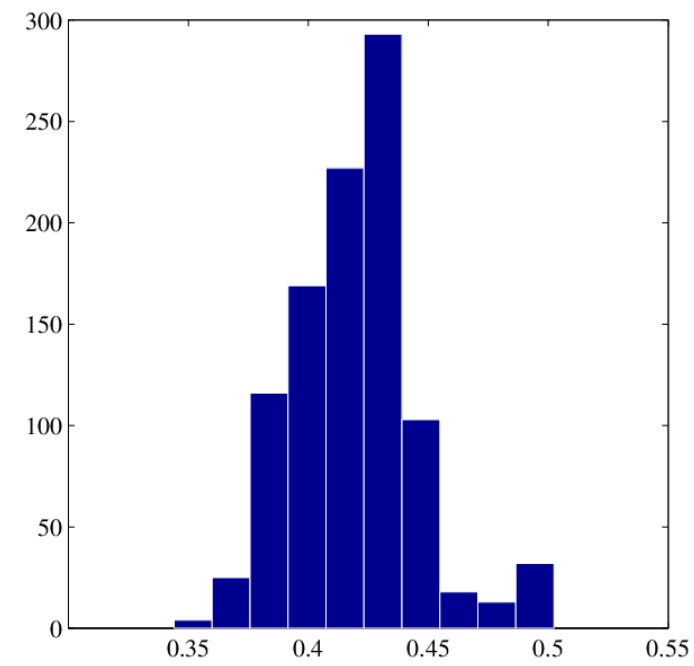

Fig. 7. Distribution of $\mathrm{E}_{\mathrm{OOB}}$ of different features.

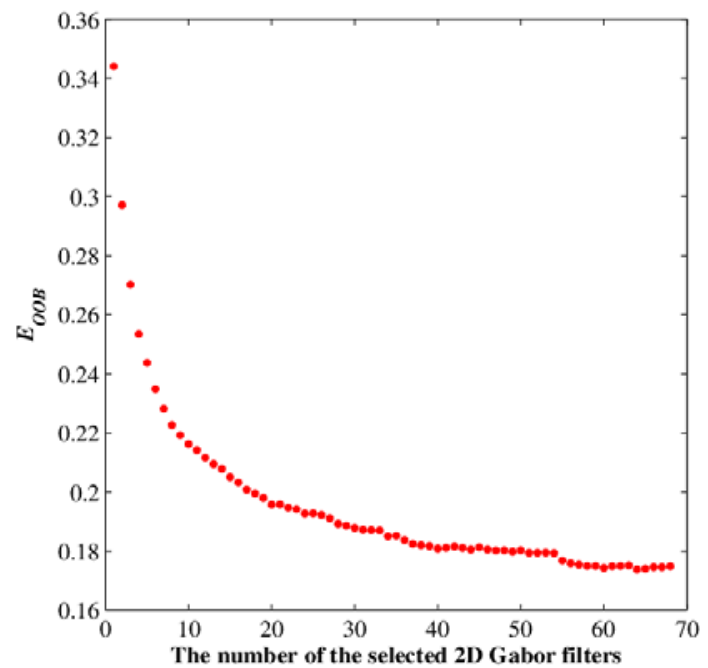

Fig. 8. Effect of Gabor filters selection. 

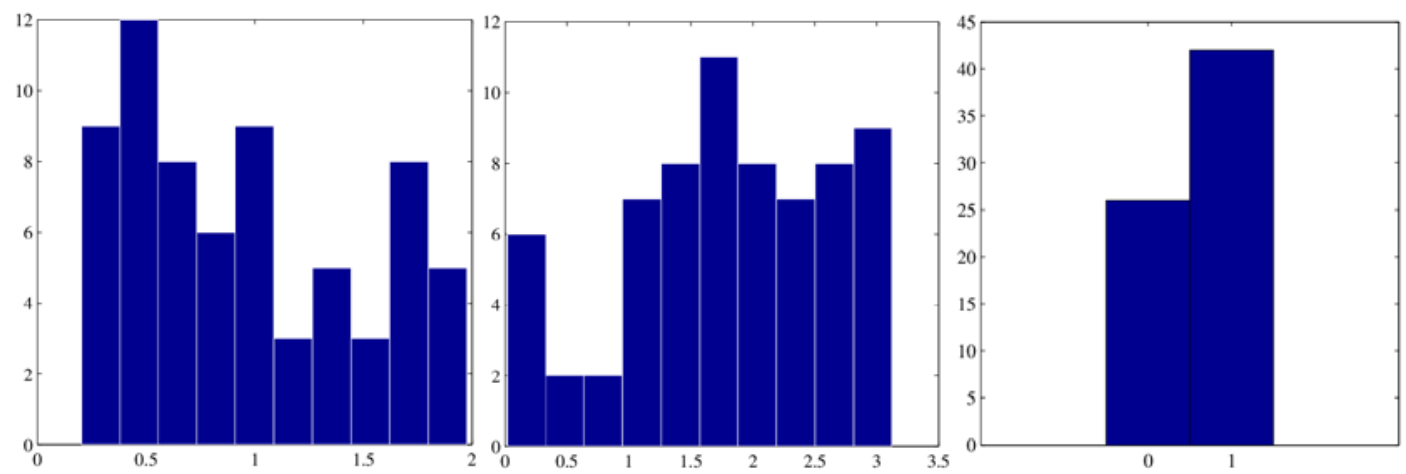

Fig. 9. Distribution of parameters $\sigma, \theta$ and $\varphi$ of the selected 2D Gabor filters.

\subsection{Comparison to prior art}

In this section, the steganalysis featue extracted by the selected 68 2D Gabor filters is compared with the four latest steganalysis features such as CC-JRM, DCTR, PHARM and GH for three content-adaptive JPEG steganography. The threshold $T$ in Eq. (8) is set to 2, then the dimensionality of the proposed steganalysis feature is 6936. The dimensionalities of different steganalysis features are shown in Table 2. Noticed that, the 68 2D Gabor filters are selected according to the detection performance for J-UNIWARD steganography with quality factor 75 and payload 0.4bpac.

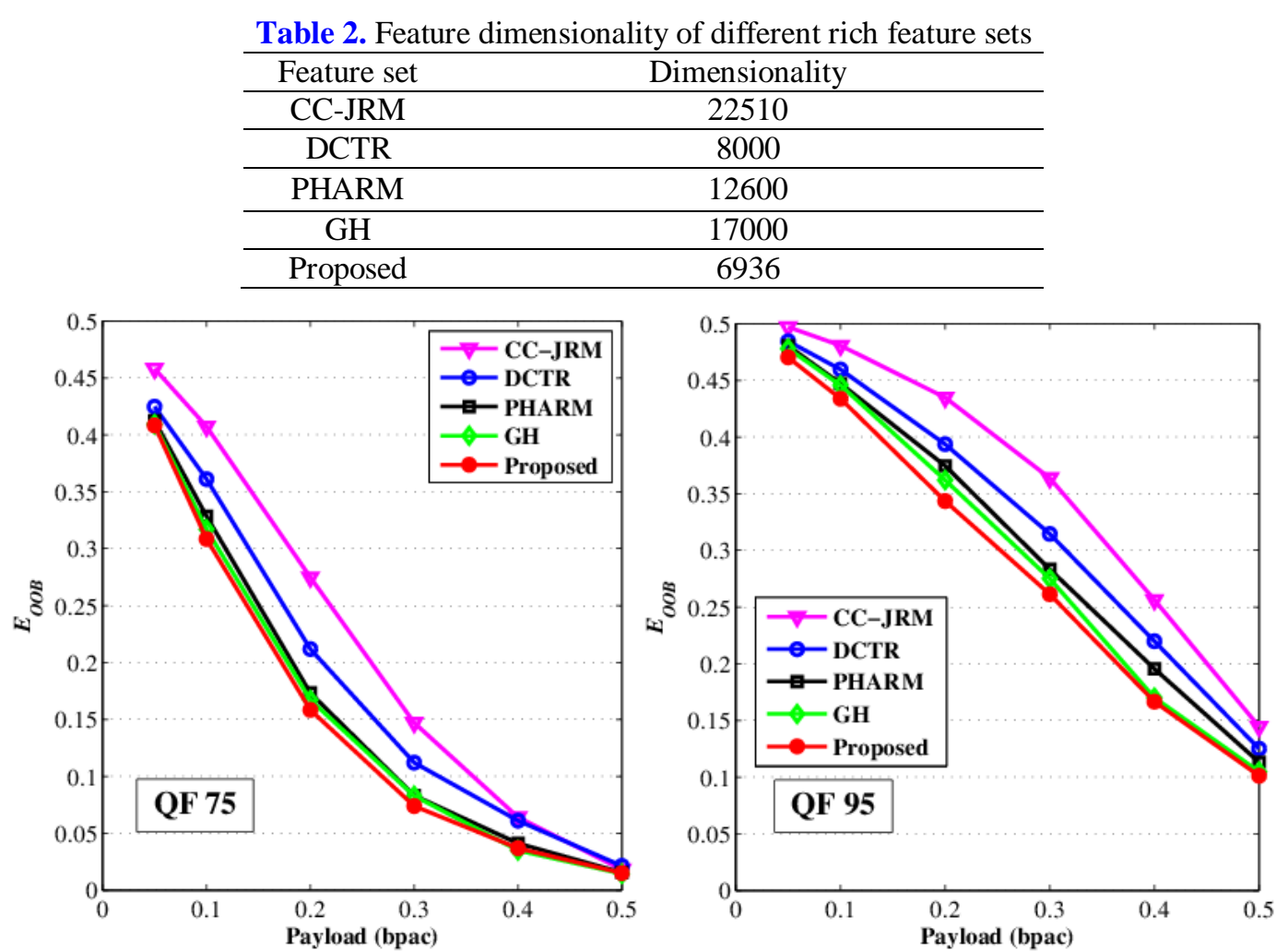

Fig. 10. Detection error $E_{O O B}$ for UED for quality factor 75 and 95 when steganalyzed with the proposed feature and the other rich feature sets. 
In Fig. 10, the detection performances of the five different steganalysis features are presented for UED steganography. From Fig. 10, it can be seen that the proposed steganalysis feature based on the selected 2D Gabor filters can achieve the best detection accuracies by comparing with the other steganalysis features. For example, when quality factor is 75 and payload is $0.2 \mathrm{bpac}$, the $\mathrm{E}_{\mathrm{OOB}}$ of CC-JRM, DCTR, PHARM, GH and the proposed steganalysis features are $28.48,21.18 \%, 17.32 \%, 16.71 \%$ and $15.83 \%$ respectively. At the same time, the feature dimensionality of the proposed steganalysis feature is lowest. When quality factor is 95 , the detection accuracies of the proposed steganalysis feature are also best. In summary, for UED steganography, not only the feature dimensionality can be reduced but also the detection performances can be improved by selecting the 2D Gabor filters.
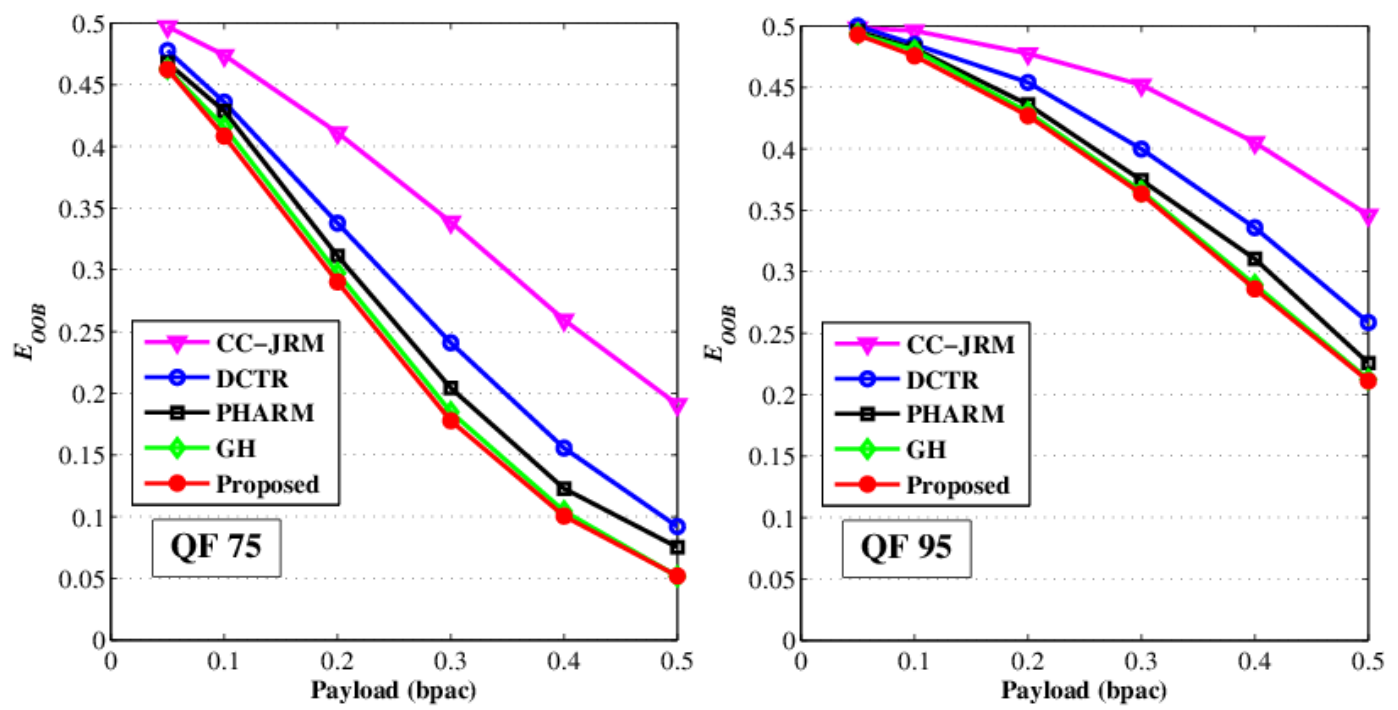

Fig. 11. Detection error $E_{O O B}$ for J-UNIWARD for quality factor 75 and 95 when steganalyzed with the proposed feature and the other rich feature sets.
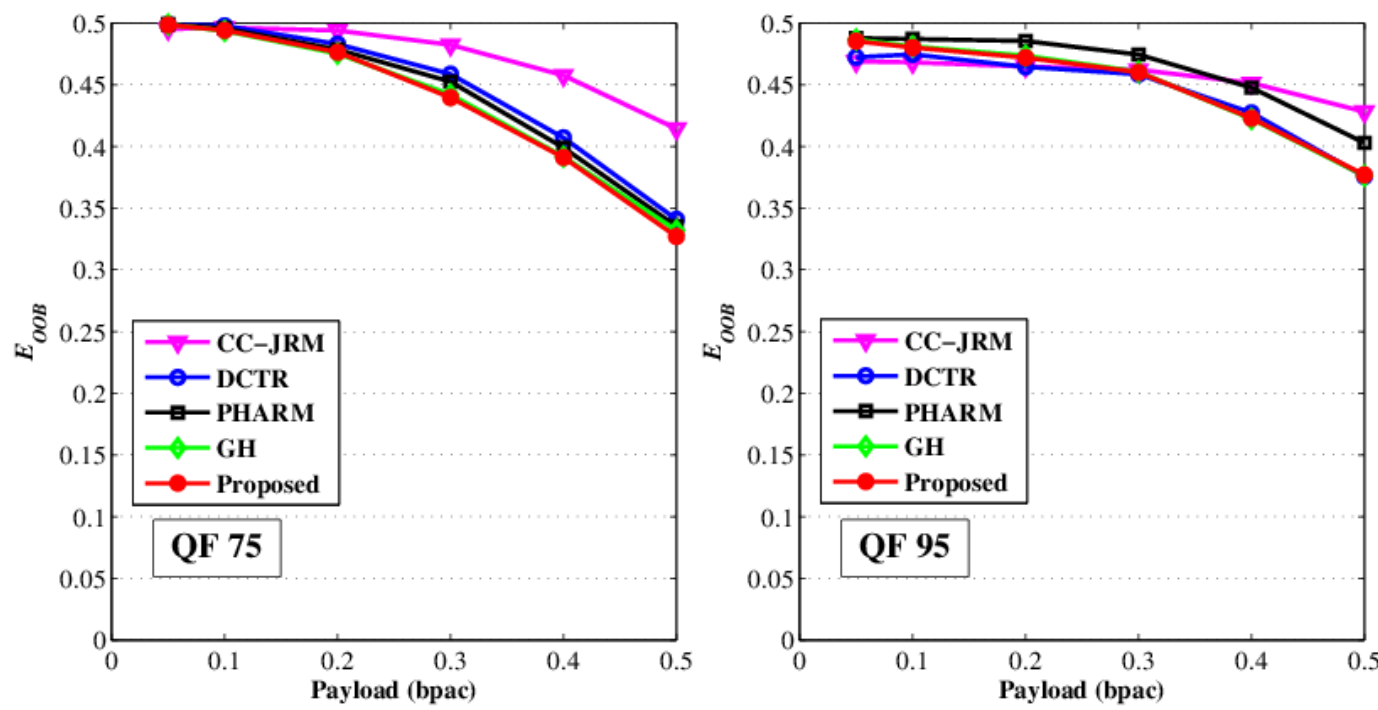

Fig. 12. Detection error $E_{O о B}$ for SI-UNIWARD for quality factor 75 and 95 when steganalyzed with the proposed feature and the other rich feature sets. 
In Fig. 11, the detection performances of the different steganalysis features are given for J-UNIWARD steganography. From Fig. 11, it can be seen that the proposed steganalysis feature can achieve the best detection performance for J-UNIWARD when quality factor is 75 . For example, in contrast to CC-JRM, DCTR, PHARM and GH, the $\mathrm{E}_{\text {Oов }}$ can be improved by $12.09 \%, 4.77 \%, 2.12 \%$ and $0.72 \%$ when payload is 0.2 bpac. When quality factor is 95 , the detection accuracy of the proposed steganalysis feature is approximate with GH while the feature dimensionality is reduced.

In Fig. 12, the detection performances of the different steganalysis features are given for SI-UNIWARD steganography. From Fig. 12, it can be seen that the proposed steganalysis feature can achieve the approximate detection performances with GH. However, the feature dimensionality of the proposed steganalysis feature is low.

In Table 3 and Table 4, the false alarm (FA) and missed detection (MD) are also presetented for different JPEG steganography algorithms, payloads and quality factors. From the Table 3 and Table 4, it can be seen that the false alarm and missed detection are approximate.

Table 3. False alarm and missed detection of different feature sets when QF is 75

\begin{tabular}{|c|c|c|c|c|c|c|c|c|c|c|c|}
\hline \multirow{2}{*}{ Stegography } & \multirow{2}{*}{ Payload } & \multicolumn{2}{|c|}{ CC-JRM } & \multicolumn{2}{|c|}{ DCTR } & \multicolumn{2}{|c|}{ PHARM } & \multicolumn{2}{|c|}{ GH } & \multicolumn{2}{|c|}{ Proposed } \\
\hline & & FA & $\mathrm{MD}$ & FA & MD & FA & MD & FA & MD & FA & MD \\
\hline \multirow{6}{*}{ UED } & 0.05 & 0.4582 & 0.4573 & 0.4248 & 0.4255 & 0.4125 & 0.4127 & 0.4094 & 0.4097 & 0.4083 & 0.4076 \\
\hline & 0.10 & 0.4073 & 0.4081 & 0.3611 & 0.3605 & 0.3285 & 0.3298 & 0.3170 & 0.3173 & 0.3085 & 0.3068 \\
\hline & 0.20 & 0.2838 & 0.2858 & 0.2111 & 0.2125 & 0.1712 & 0.1752 & 0.1671 & 0.1671 & 0.1565 & 0.1601 \\
\hline & 0.30 & 0.1471 & 0.1470 & 0.1121 & 0.1132 & 0.0831 & 0.0823 & 0.0828 & 0.0821 & 0.0739 & 0.0755 \\
\hline & 0.40 & 0.0641 & 0.0639 & 0.0611 & 0.0603 & 0.0411 & 0.0400 & 0.0350 & 0.0348 & 0.0368 & 0.0367 \\
\hline & 0.50 & 0.0181 & 0.0179 & 0.0213 & 0.0221 & 0.0155 & 0.0153 & 0.0144 & 0.0144 & 0.0150 & 0.0150 \\
\hline \multirow{6}{*}{ J-UNIWARD } & 0.05 & 0.4972 & 0.4973 & 0.4776 & 0.4755 & 0.4677 & 0.4675 & 0.4629 & 0.4621 & 0.4624 & 0.4628 \\
\hline & 0.10 & 0.4739 & 0.4735 & 0.4359 & 0.4362 & 0.4285 & 0.4283 & 0.4158 & 0.4138 & 0.4083 & 0.4089 \\
\hline & 0.20 & 0.4111 & 0.4110 & 0.3379 & 0.3371 & 0.3114 & 0.3117 & 0.2974 & 0.2968 & 0.2902 & 0.2977 \\
\hline & 0.30 & 0.3387 & 0.3385 & 0.2409 & 0.2403 & 0.2042 & 0.2044 & 0.1847 & 0.1878 & 0.1757 & 0.1723 \\
\hline & 0.40 & 0.2594 & 0.2597 & 0.1555 & 0.1544 & 0.1227 & 0.1229 & 0.1044 & 0.1072 & 0.1004 & 0.1001 \\
\hline & 0.50 & 0.1958 & 0.1953 & 0.0920 & 0.0911 & 0.0753 & 0.0750 & 0.0516 & 0.0532 & 0.0519 & 0.0517 \\
\hline \multirow{6}{*}{ SI-UNIWARD } & 0.05 & 0.4951 & 0.4947 & 0.4990 & 0.4978 & 0.4990 & 0.4976 & 0.4988 & 0.4980 & 0.4985 & 0.4974 \\
\hline & 0.10 & 0.4962 & 0.4933 & 0.4977 & 0.4956 & 0.4956 & 0.4953 & 0.4936 & 0.4931 & 0.4940 & 0.4923 \\
\hline & 0.20 & 0.4939 & 0.4954 & 0.4829 & 0.4833 & 0.4785 & 0.4766 & 0.4751 & 0.4755 & 0.4763 & 0.4770 \\
\hline & 0.30 & 0.4823 & 0.4811 & 0.4586 & 0.4543 & 0.4525 & 0.4539 & 0.4421 & 0.4411 & 0.4395 & 0.4377 \\
\hline & 0.40 & 0.4576 & 0.4574 & 0.4070 & 0.4075 & 0.3989 & 0.3974 & 0.3916 & 0.3920 & 0.3911 & 0.3989 \\
\hline & 0.50 & 0.4143 & 0.4123 & 0.3410 & 0.3411 & 0.3353 & 0.3350 & 0.3320 & 0.3310 & 0.3270 & 0.3243 \\
\hline
\end{tabular}

Table 4. False alarm and missed detection of different feature sets when QF is 95

\begin{tabular}{|c|c|c|c|c|c|c|c|c|c|c|c|}
\hline \multirow{2}{*}{ Stegography } & \multirow{2}{*}{ Payload } & \multicolumn{2}{|c|}{ CC-JRM } & \multicolumn{2}{|c|}{ DCTR } & \multicolumn{2}{|c|}{ PHARM } & \multicolumn{2}{|c|}{$\mathrm{GH}$} & \multicolumn{2}{|c|}{ Proposed } \\
\hline & & FA & MD & FA & MD & FA & $\mathrm{MD}$ & FA & MD & FA & MD \\
\hline \multirow{6}{*}{ UED } & 0.05 & 0.4972 & 0.4953 & 0.4845 & 0.4821 & 0.4805 & 0.4833 & 0.4781 & 0.4744 & 0.4703 & 0.4732 \\
\hline & 0.10 & 0.4807 & 0.4825 & 0.4596 & 0.4574 & 0.4472 & 0.4449 & 0.4457 & 0.4423 & 0.4338 & 0.4333 \\
\hline & 0.20 & 0.4349 & 0.4339 & 0.3937 & 0.3907 & 0.3747 & 0.3705 & 0.3621 & 0.3645 & 0.3436 & 0.3451 \\
\hline & 0.30 & 0.3638 & 0.3647 & 0.3145 & 0.3103 & 0.2833 & 0.2874 & 0.2751 & 0.2778 & 0.2614 & 0.2612 \\
\hline & 0.40 & 0.2564 & 0.2554 & 0.2199 & 0.2145 & 0.1955 & 0.1906 & 0.1700 & 0.1689 & 0.1665 & 0.1657 \\
\hline & 0.50 & 0.1445 & 0.1410 & 0.1251 & 0.1273 & 0.1132 & 0.1121 & 0.1048 & 0.1055 & 0.1012 & 0.1000 \\
\hline \multirow{6}{*}{ J-UNIWARD } & 0.05 & 0.4985 & 0.4976 & 0.4999 & 0.4956 & 0.4957 & 0.4934 & 0.4938 & 0.4956 & 0.4927 & 0.4911 \\
\hline & 0.10 & 0.4961 & 0.4973 & 0.4850 & 0.4821 & 0.4816 & 0.4816 & 0.4801 & 0.4811 & 0.4756 & 0.4723 \\
\hline & 0.20 & 0.4775 & 0.4770 & 0.4541 & 0.4577 & 0.4360 & 0.4341 & 0.4297 & 0.4274 & 0.4269 & 0.4259 \\
\hline & 0.30 & 0.4520 & 0.4501 & 0.3999 & 0.3967 & 0.3745 & 0.3700 & 0.3655 & 0.3623 & 0.3634 & 0.3644 \\
\hline & 0.40 & 0.4051 & 0.4023 & 0.3358 & 0.3327 & 0.3105 & 0.3145 & 0.2893 & 0.2887 & 0.2860 & 0.2853 \\
\hline & 0.50 & 0.3461 & 0.3412 & 0.2587 & 0.2583 & 0.2257 & 0.2278 & 0.2124 & 0.2143 & 0.2111 & 0.2089 \\
\hline \multirow{6}{*}{$\begin{array}{c}\text { SI-UNIWAR } \\
\text { D }\end{array}$} & 0.05 & 0.4688 & 0.4672 & 0.4722 & 0.4735 & 0.4877 & 0.4843 & 0.4866 & 0.4887 & 0.4852 & 0.4842 \\
\hline & 0.10 & 0.4682 & 0.4653 & 0.4746 & 0.4741 & 0.4870 & 0.4866 & 0.4810 & 0.4811 & 0.4801 & 0.4778 \\
\hline & 0.20 & 0.4648 & 0.4666 & 0.4645 & 0.4666 & 0.4854 & 0.4812 & 0.4736 & 0.4732 & 0.4719 & 0.4705 \\
\hline & 0.30 & 0.4621 & 0.4621 & 0.4585 & 0.4578 & 0.4745 & 0.4733 & 0.4605 & 0.4600 & 0.4600 & 0.4653 \\
\hline & 0.40 & 0.4514 & 0.4500 & 0.4272 & 0.4268 & 0.4476 & 0.4487 & 0.4223 & 0.4241 & 0.4228 & 0.4221 \\
\hline & 0.50 & 0.4281 & 0.4275 & 0.3757 & 0.3742 & 0.4029 & 0.4027 & 0.3762 & 0.3753 & 0.3770 & 0.3778 \\
\hline
\end{tabular}

From all the above comparisons with other rich feature sets, it can be found that the 2D Gabor filters selection is necessary for steganalysis of content-adaptive JPEG steganography. 
By selecting the 2D Gabor filters, the feature dimensionality can be reduced and the detection performances also can be improved to some extent.

\section{Conclusion}

2D Gabor filter can achieve optimal joint localization properties in the spatial domain and in the spatial frequency domain. It can capture the embedding changes in texture regions caused by content-adaptive JPEG steganography effectively. Therefore, the steganalysis feature based on 2D Gabor filters with different scale and orientation parameters can achieve a competitive detection performance. However, the feature dimensionality and time consuming of the current steganalysis method based on 2D Gabor filters is not satisfactory, so a new steganalysis method is proposed by selecting the optimal 2D Gabor filters . The experimental results show that the proposed method is effective. Note that, in this paper, the selection strategy for 2D Gabor filter is greedy and it is time-consuming and is not the best choice. The better selection strategy should be explored in the future study. In addition, the impact of steganography algorithms, quality factor and so on for Gabor filter selection also should be explored in the future.

\section{References}

[1] D. Upham. Steganographic algorithm JSteg. Software available at http://zooid.org/paul/crypto/jsteg/.

[2] P. Sallee, "Model-based methods for steganography and steganalysis," International Journal of Image Graphics, vol. 5, no. 1, pp. 167-190, January, 2005. Article (CrossRef Link)

[3] N. Provos, "Defending against statistical steganalysis," in Proc. of 10th Usenix Security Symposium, pp. 323-335, August 13-17, 2001. Article (CrossRef Link).

[4] A. Westfeld, "High capacity despite better steganalysis (F5-A steganographic algorithm)," in Proc. of 4th International. Workshop on Information Hiding, pp. 289-302, April 25-27, 2001. Article (CrossRef Link).

[5] J. Fridrich, T. Pevný, J. Kodovský, "Statistically undetectable JPEG steganography: dead ends, challenges, and opportunities," in Proc. of 9th ACM Multimedia and Security Workshop, pp. 3-14, September 20-21, 2007. Article (CrossRef Link).

[6] J. Fridrich, M. Goljan, and D. Soukal, "Perturbed Quantization Steganography,” ACM Multimedia System Journal, vol.11, no.2, pp.98-107, February, 2005. Article (CrossRef Link).

[7] Y. Kim, Z. Duric, D. Richards, "Modified matrix encoding technique for minimal distortion steganography," in Proc. of 8th International Workshop Information Hiding, pp. 314-327, July 10-12, 2006. Article (CrossRef Link).

[8] J. Fridrich, T. Filler, "Design of adaptive steganographic schemes for digital images," in Proc. of SPIE, Electronic Imaging, Media Watermarking, Security and Forensics of Multimedia XIII," pp. OF 1-14, January 23-26, 2011. Article (CrossRef Link).

[9] F. J. Huang, J. W. Huang, Y. Q. Shi, "New Channel Selection Rule for JPEG Steganography," IEEE Transactions on Information Forensics and Security, vol. 7, no. 4, pp. 1181-1191, August, 2012. Article (CrossRefLink)

[10] C. Wang, J. Q. Ni, “An efficient JPEG steganographic scheme based on the block-entropy of DCT coefficients,” in Proc. of IEEE ICASSP, pp.1785-1788, March 25-30, 2012.

Article (CrossRef Link).

[11] L. J. Guo, J. Q. Ni, Y. Q. Shi, "An efficient JPEG steganographic scheme using uniform embedding," in Proc. of Fourth IEEE International Workshop on Information Forensics and Security, pp.169-174, December 2-5, 2012. Article (CrossRef Link).

[12] V. Holub and J. Fridrich, "Digital image steganography using universal distortion," in Proc. of 1st ACM IH\&MMSec. Workshop, pp.59-68, June 20-22, 2013. Article (CrossRef Link). 
[13] T. Filler, J. Judas, and J. Fridrich, "Minimizing additive distortion in steganography using syndrome-trellis codes," IEEE Transactions on Information Forensics and Security, vol. 6, no. 3, pp. 920-935, September, 2011. Article (CrossRef Link).

[14] S. Lyu, H. Farid, "Steganalysis using higher-order image statistics," IEEE Transactions on Information Forensics and Security, vol. 1, no. 1, pp. 111-119, March, 2006. Article (CrossRefLink).

[15] X.Y. Luo, D.S. Wang, P. Wang and F.L. Liu, "A review on blind detection for image steganography," Signal Processing, vol. 6, no. 3, pp. 2138-2157, September, 2008. Article (CrossRef Link).

[16] H. Zong, F. L. Liu and X. Y. Luo, "Blind Image Steganalysis Based on Wavelet Coefficient Correlation,” Digital Investigation, vol. 9, no. 1, pp. 58-68, June, 2012. Article (CrossRefLink).

[17] J. Kodovský, J. Fridrich, and V. Holub, "On dangers of overtraining steganography to incomplete cover model," in Proc. of the 13th ACM Multimedia \& Security Workshop, pp. 69-76, September 29-30, 2011. Article (CrossRefLink).

[18] X. F. Song, F. L. Liu, X. Y. Luo and J. C. Lu, "Steganalysis of perturbed quantization steganography based on the enhanced histogram features," Multimedia Tools and Applications, vol. 74, no. 24, pp. 11045-11071, December, 2015. Article (CrossRefLink).

[19] J. Kodovský and J. Fridrich, "Steganalysis of JPEG images using rich models," in Proc. of SPIE, Electronic Imaging, Media Watermarking, Security, and Forensics of Multimedia XIV, pp. 0A 1-13, January 22-26, 2012. Article (CrossRefLink).

[20] V. Holub and J. Fridrich, "Random projections of residuals for digital image steganalysis," IEEE Transactions on Information Forensics and Security, 8(12):1996-2006, 2013. Article (CrossRefLink).

[21] V. Holub and J. Fridrich, "Low Complexity Features for JPEG Steganalysis Using Undecimated DCT," IEEE Transactions on Information Forensics and Security, vol. 10, no. 2, pp. 219-228, February, 2015. Article (CrossRefLink).

[22] V. Holub, J. Fridrich, "Phase-Aware Projection Model for Steganalysis of JPEG Images," in Proc. of SPIE, Electronic Imaging, Media Watermarking, Security, and Forensics of Multimedia XIV, pp. 94090T-94090T-11, February 8-12, 2015. Article (CrossRefLink).

[23] X. F. Song, F. L. Liu, C. F. Yang, X. Y. Luo and Y. Zhang, "Steganalysis of Adaptive JPEG Steganography Using 2D Gabor Filters," in Proc. of the 3rd ACM Workshop on Information Hiding and Multimedia Security, pp. 15-23, June 17-19, 2015. Article (CrossRefLink).

[24] J. Kodovský, J. Fridrich and V. Holub, "Ensemble classifiers for steganalysis of digital media," IEEE Transactions on Information Forensics and Security, vol.7, no. 2, pp. 432-444, April, 2012. Article (CrossRefLink).

[25] J. G. Daugman, "Uncertainty relation for resolution in space, spatial frequency, and orientation optimized by two-dimensional visual cortical filters," Journal of the Optical Society of America A Optics and Image Science, vol. 2, no. 7, pp. 1160-1169, July, 1985. Article (CrossRefLink).

[26] M. Idrissa, M. Acheroy, "Texture classification using Gabor filters," Pattern Recognition Letters, vol. 23, no. 9, pp. 1095-1102, July, 2002. Article (CrossRefLink).

[27] M. Li, R.C. Staunton, "Optimum Gabor filter design and local binary patterns for texture segmentation,” Pattern Recognition Letters, vol. 29, no. 5, pp. 664-672, April, 2008. Article (CrossRefLink).

[28] Z. N. Sun, T. N. Tan, "Ordinal Measures for Iris Recognition," IEEE Transactions on Pattern Analysis and Machine Intelligence, vol. 31, no. 12, pp. 2211-2226, December, 2009. Article (CrossRefLink).

[29] Z. H. Chai, Z. N Sun, H. M. Vazquez, R. He and T. N. Tan, "Gabor Ordinal Measures for Face Recognition," IEEE Transactions on Information Forensics and Security, vol. 9, no. 1, pp. 14-26, January, 2014. Article (CrossRefLink).

[30] T. Denemark, V. Sedighi, V. Holub and R. Cogranne, "Selection-Channel-Aware Rich Model for Steganalysis of Digital Images," in Proc. of the 5th IEEE International Workshop on Information Forensics and Security, pp. 48-53, December 3-5, 2014. Article (CrossRefLink). 
[31] S. E. Grigorescu, N. Petkov and P. Kruizinga, “Comparison of Texture Features Based on Gabor Filters,” IEEE Transactions on Image Processing, vol. 11, no. 10, pp. 1160-1167, October, 2002. Article (CrossRefLink).

[32] P. Bas, T. Filler and T. Pevný, "Break our steganographic system-The ins and outs of organizing BOSS," in Proc. of the 13th International workshop on Information Hiding, pp. 59-70, May 18-20, 2011. Article (CrossRefLink).

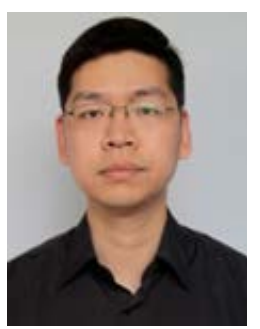

Xiaofeng Song received the B.S. degree from the School of Information and Technology, Zhengzhou University, Zhengzhou, China, in 2002, and M.S. degree from the School of Computer Science, Xidian University, Xi'an, China, in 2009. Now, he is a $\mathrm{PhD}$ Candidates of the State Key Laboratory of Mathematical Engineering and Advanced Computing, Zhengzhou Science and Technology Institute, China. His current research interest includes steganography, steganalysis and digital image forensic.

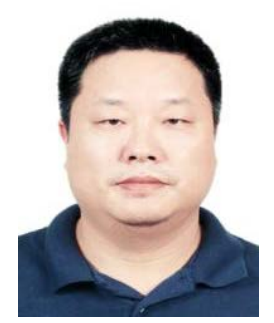

Fenlin Liu received the B.S. degree from Zhengzhou Information Science and Technology Institute, Zhengzhou, China, in 1986, the M.S. degree from Harbin Institute of Technology, Harbin, Heilongjiang, in 1992, and the Ph.D. degree from the Northeast University, Shenyang, Liaoning, in 1998. He is currently a professor of State Key Laboratory of Mathematical Engineering and Advanced Computing, and Zhengzhou Science and Technology Institute. His research interests include information hiding and security theory. He is the author or co-author of more than 100 refereed international journal and conference papers..

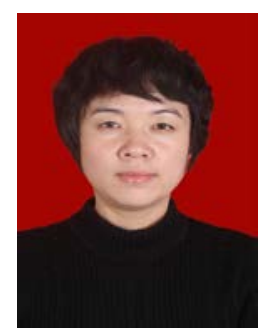

Liju Chen was born in Shannxi Province in 1973. She received the Ph. D. degree in physics at the state key Laboratory of Transient Optics and photonics, Xi'an Institute of Optics and Precision Mechanics, CAS in 2011. Her main research interests include Information Security, Communications Privacy and optical information processing.

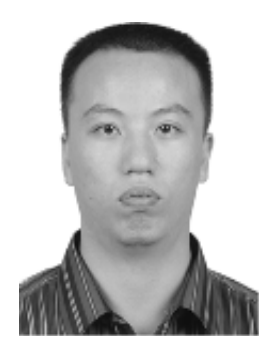

Chunfang Yang received the M.S. degree and Ph.D. degree from Zhengzhou Information Science and Technology Institute, Zhengzhou, China, in 2008 and 2012, respectively. Currently, he is a lecturer of Zhengzhou Science and Technology Institute. $\mathrm{He}$ is the author or co-author of more than 40 refereed international journal and conference papers. His research interest includes image steganography and steganalysis technique.

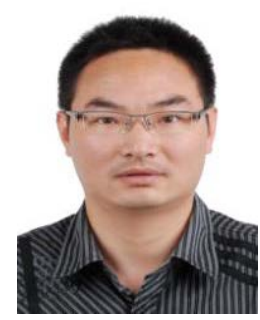

Xiangyang Luo received the M.S. degree and the Ph.D. degree from Zhengzhou Information Science and Technology Institute, Zhengzhou, China, in 2004 and 2010, respectively. He is the author or co-author of more than 70 refereed international journal and conference papers. He is currently an associate professor of State Key Laboratory of Mathematical Engineering and Advanced Computing, and Zhengzhou Science and Technology Institute. In addition, he also recently served as guest editor of some special issues of International Journal "Multimedia Tools and Applications" and "International Journal of Internet". His research interest includes multimedia security, image steganography/steganalysis. 